\title{
IL-18 modulates chronic fungal asthma in a murine model; putative involvement of Toll-like receptor-2
}

\author{
K. Blease, S.L. Kunkel and C.M. Hogaboam \\ Department of Pathology, University of Michigan Medical School, 1301 Catherine Road, Ann Arbor MI, 48109-0602, USA, \\ Fax: ++1 734764 2397, e-mail: hogaboam@med.umich.edu
}

Received 22 March 2001; returned for revision 12 July 2001; accepted by L. G. Letts 23 July 2001

\begin{abstract}
Fungus-induced asthmatic disease is characterized by persistent airway hyperreactivity and remodeling. Objective and design: To determine the role of IL-18 in the allergic airway response to Aspergillus fumigatus conidia in a murine model of $A$. fumigatus-induced asthma.

Methods: A. fumigatus-sensitized mice were depleted of IL18 using a polyclonal anti-IL-18 antibody for 3 days after a conidia challenge.

Results: Airway hyperresponsiveness and eosinophil numbers were significantly elevated 3-30 days after conidia challenge compared to the normal serum-treated group. Histological evidence showed retention of A. fumigatus conidia, airway remodeling, subepithelial fibrosis, and increased collagen deposition in the lungs of IL-18-depleted mice at day 30 after the conidia challenge. Prolonged retention of conidia in IL-18 depleted A. fumigatus-sensitized mice was associated with decreased Toll-like receptor-2 (TLR-2) expression compared with the control group.

Conclusions: IL-18 modulates the innate immune response against $A$. fumigatus conidia and prevents the development of severe fungus-induced asthmatic disease.
\end{abstract}

Key words: Infectious immunity - Fungi - Allergy - Lung Inflammatory mediators

\section{Introduction}

Asthma is a respiratory disorder characterized by airway hyperresponsiveness (AHR) to a variety of specific and nonspecific stimuli, airway inflammation associated with upregulation of Th2 cells and cytokines, eosinophilia, subepithelial fibrosis, and excessive mucus production [1]. Sensitization of individuals to fungal antigens has become particularly prevalent in recent years, in particular, the ubiquitous fungi, Aspergillus fumigatus (A. fumigatus) [2]. Individuals with fungal allergy exhibit similar pathological characteristics to those observed in asthmatics. In contrast to patients with

Correspondence to: C. M. Hogaboam allergic bronchopulmonary aspergillosis (ABPA), subjects with fungal allergy exhibit little or no evidence of fungal growth in their lungs, despite the persistence of sensitivity to fungal antigens [3]. It is clear that the soluble mediators such as cytokines exacerbate fungus retention [3]. While the immune response to A. fumigatus antigens in allergic asthma is assumed to be the result of CD4+ Th2 cells, the effective clearance of conidia requires a $\mathrm{Th} 1$ response, associated with elevated IL-12 and IFN- $\gamma$ cytokine levels [4]. Therefore, the elucidation of allergic responses to A. fumigatus and of the anti-fungal defenses during allergic disease are of clinical interest.

IL-18 was initially described as an IFN $\gamma$-inducing factor that upregulates the IL-12R $\beta$ subunit on T cells and has generally been considered a Th1 type cytokine $[5,6]$. IL-18 promotes resolution of bacterial infection [7] and induces fungicidal activity against Cryptococcus neoformans through the production of IFN- $\gamma$ by NK cells [8]. While the innate response to fungal infection involves the coordinated action of IL-18 in the Th1 immune response, little is known about the anti-fungal role of IL-18 in the context of allergic fungal disease.

The present study was designed to define the role of IL-18 in experimental fungal asthma. The results presented herein demonstrate a clear role for IL-18 expression at early time points following conidia challenge and suggest that the IL-18 synthesis is essential for the effective clearance of conidia in the context of allergic fungal airway disease. Accordingly, immunoneutralization of IL-18 for 3 days following conidia challenge in A. fumigatus-sensitized mice leads to the retention of conidia within the airways and persistent airway hyperreactivity and peribronchial fibrosis that is prominent at $\mathbf{3 0}$ days after the conidia challenge.

\section{Materials and methods}

Sensitization of mice to A. fumigatus

Specific-pathogen free (SPF), female CBA/J mice (Jackson Laboratories; Bar Harbor, ME) were maintained in a SPF facility for the duration of this study. Prior approval for mouse usage in the development of the 
allergic fungal asthma model described herein was obtained from University Laboratory Animal Medicine facility at the University of Michigan Medical School. Sensitization of mice to a commercially available preparation of soluble A. fumigatus antigens (Greer Laboratories, Lenoir, NC) was performed as previously described in detail [9]. Briefly, mice received a total of $10 \mu \mathrm{g}$ of $A$. fumigatus antigens dissolved in $0.2 \mathrm{ml}$ of IFA (Sigma, St. Louis, MO) distributed equally between the peritoneal cavity and s.c. Two weeks later, and for three subsequent weeks, mice received a total of $20 \mu \mathrm{g}$ of A. fumigatus $\mathrm{Ag}$, dissolved in normal saline, via the intranasal route. One week after the third intranasal challenge, each mouse received $5.0 \times 10^{6}$ live A. fumigatus conidia, strain 13073 , suspended in $30 \mu$ of $0.1 \%$ Tween- 80 via the intratracheal route [10].

\section{Production of anti-IL-18 Abs and immunoneutralization of IL-18}

Rabbit anti-murine IL-18 Ab were prepared by multiple-site immunization of New Zealand White rabbits with recombinant murine IL-18 (R\&D Systems, Rochester, MN) in CFA. The polyclonal Ab was titered by direct ELISA and specifically verified by the failure to cross-react to other murine cytokines or chemokines. As a control, preimmune normal rabbit serum (NRS) was used. The endotoxin content in IL-18 antiserum and NRS was below detection level $(<0.05$ EU/ml PYROGENT; BioWhittaker, Walkersville, MD). In depletion studies, mice were pretreated with polyclonal rabbit anti-murine IL-18 antibodies $(0.5 \mathrm{ml}$, titers of $10^{6} / \mathrm{ml}$ ) given intraperitoneally $1 \mathrm{~h}$ prior to intratracheal administration of conidia and 2 days post conidia. The volume of antiserum was considered to be sufficient to neutralize systemic endogenous IL18 in that the biological half-life of the antibody was $\sim 48$ h (C.M. Hogaboam, unpublished observation). Control animals received $0.5 \mathrm{ml}$ of normal rabbit serum (NRS). Airway physiology and function was assessed 3, 7, and 30 days following conidia challenge.

\section{Measurement of bronchial hyperresponsiveness}

Immediately prior to and at days 3, 7, and 30 after the intratracheal $A$. fumigatus conidia challenge, bronchial hyperresponsiveness in A. fumigatus-sensitized mice was assessed in a Buxco ${ }^{\mathrm{TM}}$ plethysmograph (Buxco, Troy, NY) [11]. Mice were anesthetized with sodium pentobarbital (40 mg/ml i.p.; Butler Co., Columbus, $\mathrm{OH}$ ) prior to their intubation for ventilation with a Harvard pump ventilator (Harvard Apparatus, Reno, $\mathrm{NV}$ ). The following ventilation parameters were used: tidal volume = $0.25 \mathrm{ml}$, breathing frequency $=120 / \mathrm{min}$, and positive end-expiratory pressure $\sim 3 \mathrm{~cm} \mathrm{H} \mathrm{H}_{2} \mathrm{O}$. Within the sealed plethysmograph mouse chamber, transpulmonary pressure (i.e. tracheal pressure - mouse chamber pressure) and inspiratory volume or flow were continuously monitored online by an adjacent computer. Airway resistance was calculated online via computer software (Buxco, Troy, N.Y.), and was determined by the division of the transpulmonary pressure by the change in inspiratory volume. Following a baseline period in the Buxco apparatus, anesthetized and intubated mice received a dose of $1 \mu \mathrm{g}$ of methacholine by tail vein injection, and airway responsiveness to this non-selective bronchoconstrictor was again calculated online. At the conclusion of the assessment of airway responsiveness, each mouse was killed by exsanguination, and a bronchoalveolar lavage (BAL) was performed with $1 \mathrm{ml}$ of normal saline. Approximately $500 \mu \mathrm{l}$ of blood was also collected from each mouse and transferred to a microcentrifuge tube. Sera were obtained after the sample was centrifuged at $15000 \mathrm{rpm}$ for $10 \mathrm{~min}$. Whole lungs were finally dissected from each mouse and snap frozen in liquid $\mathrm{N}_{2}$ or prepared for histological analysis.

\section{Morphometric analysis of leukocyte accumulation in BAL samples}

Neutrophils, macrophages, eosinophils, and lymphocytes were quantified in bronchoalveolar lavage fluid (BALF) samples applied to coded microscope slides using a cytospin (Shandon Scientific, Runcorn, UK). Identification of each cell type in the cytospins was facilitated by differential staining with Wright-Giemsa differential stain and expressed as a percentage of total cells after counting 300 cells in $10-20$ high power fields $(1000 \times)$ per slide. A total of $1 \times 10^{6} \mathrm{BAL}$ cells were spun onto each slide to compensate for differences in cell retrieval.

\section{ELISA analysis}

Murine IL-18, MIP-2, IFN- $\gamma$, TNF- $\alpha$, eotaxin, RANTES, and IL-12 protein levels were determined in $50 \mu$ l of whole lung homogenates, using a standardized sandwich ELISA technique previously described in detail [12]. Whole lungs were homogenized in $1 \mathrm{ml}$ of normal saline containing $1 \mathrm{mg}$ of protease inhibitor $\left(\right.$ Complete $^{\mathrm{TM}}$, Boehringer Mannheim, Indianapolis, IN) with a Tissue Tearor. Recombinant murine IL-18 was used to generate the standard curves from which the concentrations present in the samples were derived. The limit of ELISA detection for IL-18 was consistently above $50 \mathrm{pg} / \mathrm{ml}$ and the ELISA was screened to ensure the specificity of each antibody used. MMP-2 activity was measured in $100 \mu \mathrm{l}$ samples of cell culture supernatants using MMP-2 ELISA activity assay system (Amersham Pharmacia Biotech, Piscataway, NJ) according to the manufacturer's directions.

\section{Whole lung histological analysis}

Whole lungs from $A$. fumigatus-sensitized mice prior to and after $A$. fumigatus conidia challenge were fully inflated by intratracheal perfusion with $4 \%$ paraformaldehyde. Lungs were then dissected and placed in fresh paraformaldehyde for $24 \mathrm{~h}$. Routine histological techniques were used to paraffin-embed this tissue, and 5- $\mathrm{mm}$ sections of whole lung were stained with hematoxylin and eosin, Masson trichrome, Periodic Acid Schiff (PAS), and Gomori methanamine silver (GMS).

\section{Preparation of $c D N A$ and reverse transcriptase (RT)-PCR amplification}

Total RNA samples were prepared from whole lung samples removed from A. fumigatus sensitized mice at days $0,3,7$, and 30 after conidia challenge. RNA from specific samples was reverse transcribed into cDNA utilizing a BRL reverse transcription kit and oligo (dT) 12-18 primer. The amplification solution contained $50 \mathrm{mM} \mathrm{KCl}, 10 \mathrm{mM}$ Tris-

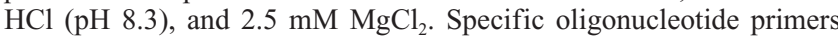
were added ( $200 \mathrm{ng} / \mathrm{sample})$ to the buffer, along with $5 \mu \mathrm{l}$ of reverse transcribed cDNA sample. The following oligonucleotide primers were used: TLR2 primer sequences: sense, 5'CTCAGGATAGGAAATGTAGAGACT 3'; antisense, 5'AATCGCCGAGCCCATTGAGGGTA 3', 426-base-pair (bp) product. $\beta$-actin primer sequences: sense, $5^{\prime} \mathrm{GC}$ TCGGCCGTGGTGGTGAAGC 3'; antisense, 5'GTGGGGCGCCCCAGGCACCA 3', 450-bp product.

The cDNA was amplified using the following cycling parameters: the mixture was first incubated for $4 \mathrm{~min}$ at $94^{\circ} \mathrm{C}$ and then cycled 32 times at $94^{\circ} \mathrm{C}$ for $45 \mathrm{sec}, 55^{\circ} \mathrm{C}$ for $45 \mathrm{sec}$, and elongated at $72^{\circ} \mathrm{C}$ for $45 \mathrm{sec}$. After amplification the samples were separated on a $2 \%$ agarose gel containing $0.3 \mu \mathrm{g} / \mathrm{ml}$ of ethidium bromide, and bands were visualized and photographed using a translucent UV source.

\section{Statistical analysis}

All results are expressed as mean \pm standard error of the mean (SEM) from 2 repeated studies. Analysis of variance (ANOVA) and Dunnett's test for multiple comparisons were used to determine statistical significance in both groups at various times after conidia challenge; $\mathrm{P}<0.05$ was considered statistically significant. 


\section{Results}

IL-18 levels are elevated in whole lung following conidia challenge in A. fumigatus-sensitized mice

To determine the temporal expression of IL-18 in A. fumigatus-sensitized mice following conidia challenge, IL-18 levels in whole lung homogenates were measured by ELISA. IL-18 levels measured in whole lung homogenates from naive mice were increased following $A$. fumigatus sensitization prior to conidia challenge $(10.1 \pm 1.2$ to $18.0 \pm 2.4 \mathrm{ng} / \mathrm{mg}$ protein). IL-18 was significantly elevated above these levels at day 3 $(\mathrm{P}<0.05 ; 26.8 \pm 4 \mathrm{ng} / \mathrm{mg}$ protein) after conidia challenge (Fig. 1), however, levels returned to baseline after day 3 . Since IL-18 levels were significantly elevated up to day 3 after conidia, anti-IL-18 antiserum or normal rabbit serum was administered to mice from days 0 to 3 after conidia. AntiIL-18 antiserum effectively attenuated IL-18 levels compared with NRS controls (Fig. 1).

\section{IL-18 immunoneutralization does not alter IL-12 or IFN- $\gamma$} levels in whole lung homogenates from allergic mice

IL-18 has been shown to induce the expression and release of IFN- $\gamma$ from T cells and macrophages in combination with IL12 [8]. Therefore, to determine the effect of IL-18 immunoneutralization on the levels of IFN- $y$ and IL-12, both cytokines were measured via ELISA in whole lung homogenates from A. fumigatus-sensitized mice at $0,3,7$, and 30 days after intratracheal conidia challenge in mice treated with either NRS or polyclonal anti-IL-18 Abs for the first 3 days after conidia challenge (Figs. 2A and B). Significantly elevated levels of IFN- $\gamma(\mathrm{P}<0.01)$ and IL-12 $(\mathrm{P}<0.05)$ were measured in whole lung homogenates 3 days after conidia challenge in A. fumigatus-sensitized mice, however, by day 7 both cytokines were detected at levels similar to those measured at day 0. Following immunoneutralization of IL-18 at day $0-3$ following intratracheal conidia administration, no

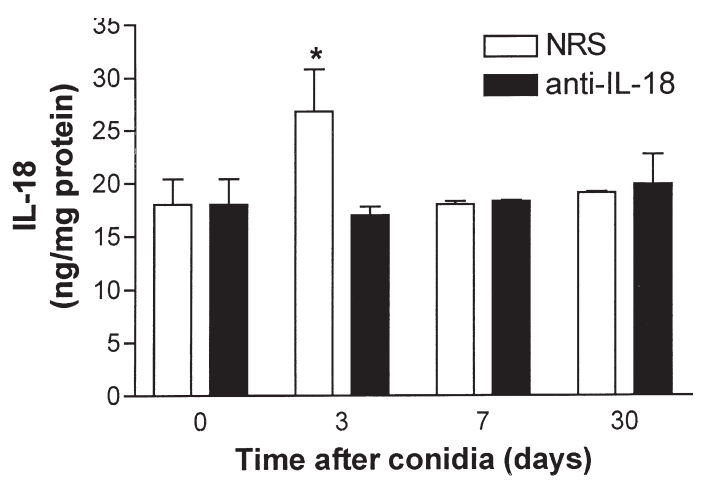

Fig. 1. IL-18 levels in whole lung homogenates from A. fumigatus-sensitized mice prior to, and at 3, 7, or 30 days post, intra-tracheal conidia challenge measured by a specific ELISA. Mice were treated with NRS (solid circles) or polyclonal anti-IL-18 antibodies (filled circles) $1 \mathrm{~h}$ prior to conidia challenge and 2 days post challenge. Results show mean \pm SEM from 5 mice at each time point. $* \mathrm{P}<0.05$ denotes significant induction of IL-18 production compared to levels measured prior to conidia challenge, $\mathrm{t}=0$.
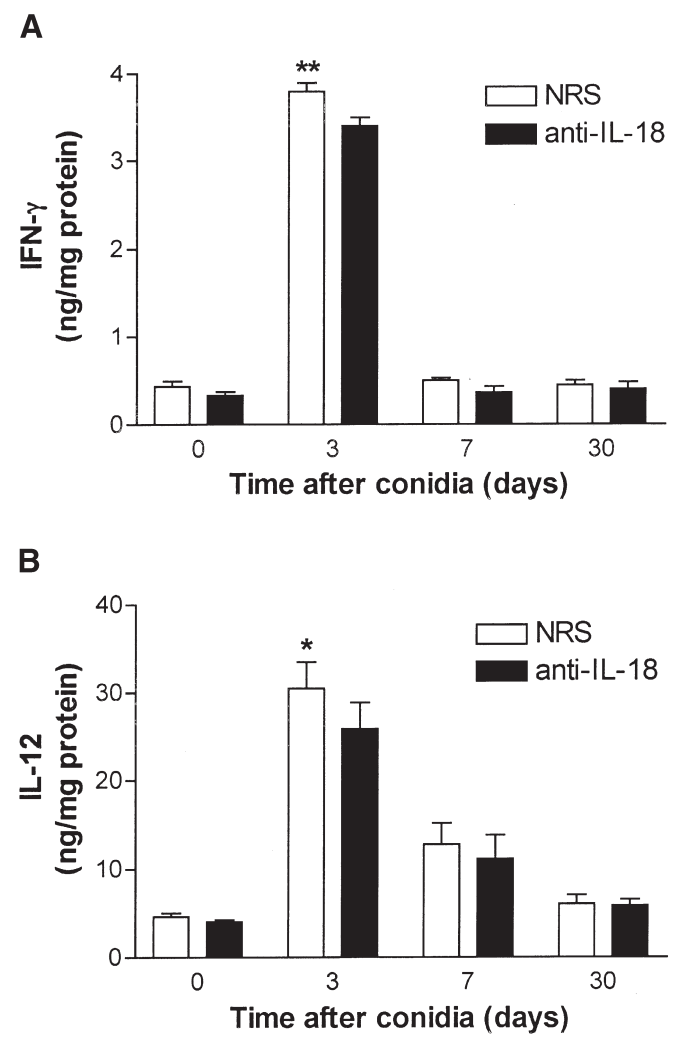

Fig. 2. IFN- $\gamma$ (A) or IL-12 (B) levels in whole lung homogenates from A. fumigatus-sensitized mice prior to, and at 3, 7, or 30 days post, intratracheal conidia challenge measured by a specific ELISA. Mice were treated with NRS (solid circles) or polyclonal anti-IL-18 antibodies (filled circles) $1 \mathrm{~h}$ prior to conidia challenge and 2 days post challenge. Results show mean \pm SEM from 5 mice at each time point. $* \mathrm{P}<0.05$ or $* * \mathrm{P}<0.01$ denotes significant induction of IFN- $\gamma$ or IL-12 production compared to levels measured prior to conidia challenge, $\mathrm{t}=0$.

significant differences in IFN- $\gamma$ (Fig. 2A) or IL-12 (Fig. 2B) levels were detected between the two groups. This data demonstrates that IL-18 immunoneutralization does not affect whole lung levels of IL-12 or IFN- $y$ after conidia challenge in A. fumigatus-sensitized mice.

\section{IL-18 neutralization alters eosinophil and neutrophil recruitment in the airways of $A$. fumigatus-sensitized mice}

Leukocytes accumulating in the lung during allergic asthma play multiple roles in the pathogenesis of the disease. However, these cells also exert a major effect on the clearance of airborne pathogens. For example, whereas eosinophils have been shown to exacerbate fungal allergic disease $[2,9,13]$, neutrophils recruited to the lung during fungal infection play a key role in early killing of the fungal conidia [3]. Therefore, leukocyte recruitment into the lung following conidia challenge was monitored for up to 30 days in A. fumigatus-sensitized mice treated with NRS or polyclonal anti-IL-18 Abs between days $0-3$.

The percentage of neutrophils in BALs from A. fumigatus-sensitized mice increased significantly 3 days after conidia challenge (Fig. $3 \mathrm{~A}$ ). Following immunoneutralization of 
A

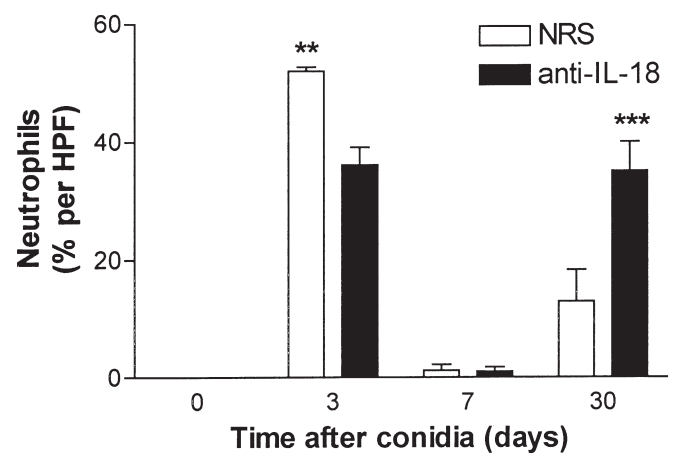

B

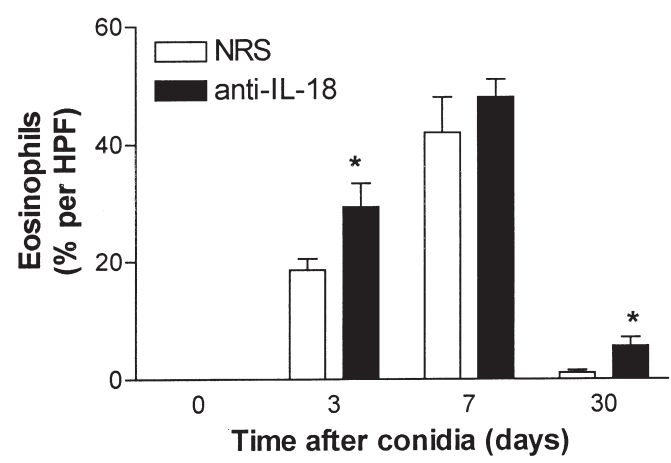

Fig. 3. Neutrophil (A) and eosinophil (B) counts in BAL samples from A. fumigatus-sensitized mice treated before, and at various times after, A. fumigatus conidia challenge. Mice were treated $1 \mathrm{~h}$ prior to, or 2 days after, conidia challenge with NRS (solid circles) or anti-IL-18 polyclonal antibodies (open circles). BAL cells were dispersed onto microscope slides using a cytospin; neutrophils and eosinophils were differentially stained with Wright-Giesma stain. A minimum of 15 high powered fields or 300 cells were examined in each cytospin. A total of $1 \times 10^{6}$ BAL cells were cytospun onto each slide to compensate for differences in cell retrieval from each mouse. Values are expressed as mean \pm SEM of the percentage of cells based total cells counted/sample. ${ }^{*} \mathrm{P}<0.05$ or $* * \mathrm{P}<0.01$ denotes significant differences in neutrophil or eosinophil numbers compared to NRS treated-mice at the same time point.

IL-18, significantly $(\mathrm{P}<0.05)$ fewer neutrophils were counted in recovered BAL at day 3 (Fig. $3 \mathrm{~A}$ ), while neutrophils were absent at day 7 in both groups. In contrast, 30 days after conidia challenge in anti-IL-18-treated mice, neutrophil numbers were significantly increased compared to NRStreated controls.

Eosinophil numbers within the BAL were elevated in NRS and anti-IL-18-treated mice at days 3 and 7 after conidia challenge, though significantly greater numbers were counted in the BALs from anti-IL-18-treated mice (Fig. 3B). Thirty days following conidia challenge, eosinophils were not present in BAL from NRS-treated controls, however, eosinophil numbers remained significantly elevated $(\mathrm{P}<0.05)$ in anti-IL-18-treated mice at this time point. Taken together, this data suggested that immunoneutralization of IL-18 causes significant alterations in the recruitment of both neutrophils and eosinophils to the allergic airway.
Table 1

\begin{tabular}{lll}
\hline & NRS & Anti-IL-18 antiserum \\
\hline MIP-2 & & \\
Day 0 & $0.4 \pm 0.01$ & $0.4 \pm 0.05$ \\
Day 3 & $2.4 \pm 0.9^{*}$ & $2.5 \pm 0.2^{*}$ \\
TNF- $\alpha$ & & \\
Day 0 & $0.3 \pm 0.03$ & $0.5 \pm 0.03$ \\
Day 3 & $3.7 \pm 0.8^{*}$ & $3.9 \pm 0.08^{*}$ \\
Eotaxin & & \\
Day 0 & $0 \pm 0$ & $0 \pm 0$ \\
Day 3 & $0.02 \pm 0.01$ & $0.04 \pm 0.01$ \\
\hline
\end{tabular}

MIP-2, TNF- $\alpha$, or eotaxin levels in whole lung homogenates from $A$. fumigatus-sensitized mice prior to, and at 3 days post, intra-tracheal conidia challenge measured by a specific ELISA. Mice were treated with NRS or polyclonal anti-IL-18 antibodies $1 \mathrm{~h}$ prior to conidia challenge and 2 days post challenge. Results show mean \pm SEM from 5 mice at each time point. ${ }^{*} \mathrm{P}<0.05$ denotes significant induction of cytokine or chemokine production compared to levels measured prior to conidia challenge, $\mathrm{t}=0$.

\section{IL-18 neutralization does not alter MIP-2, TNF- $\alpha$, or eotaxin release 3 days after conidia challenge}

To determine the role of cytokine and chemokine release 3 days after conidia challenge on leukocyte accumulation at this time point, levels of cytokines and chemokines that are involved in the recruitment of eosinophils and neutrophils, such as eotaxin, MIP-2, RANTES, and TNF- $\alpha$, were measured. Three days after conidia challenge in A. fumigatussensitized mice, significantly elevated levels of MIP-2 and TNF- $\alpha$ were observed (Table 1). However, no significant difference in the whole lung levels of these cytokines were denoted between NRS and anti-IL-18-treated mice at this time point (Table 1). In contrast, eotaxin and RANTES levels in whole lung homogenates were not significantly elevated 3 days after conidia challenge, and anti-IL-18 antiserum treatment did not further change the levels of these chemokines measured in the lung (Table 1). This data demonstrates that IL-18 immunoneutralization does not affect whole lung levels of MIP-2, TNF- $\alpha$, or eotaxin after conidia challenge in A. fumigatus-sensitized mice.

\section{Neutralization of IL-18 exacerbates airway hyperresponsiveness}

Following intratracheal challenge with live A. fumigatus conidia, A. fumigatus-sensitized mice exhibit chronic AHR to intravenously administered methacholine up to 30 days after conidia challenge, as demonstrated in previous studies [14-16]. Studies utilizing IL-18 deficient mice or IL-18 immunoneutralization methods have shown exacerbation of AHR in several acute allergic murine models in the absence of IL-18 $[17,18]$. We have also shown that the eosinophil has a prominent role in the acute AHR to soluble A. fumigatus antigen challenge [9]. To assess the role of IL-18 immunoneutralization on AHR in the present study, intravenous administration of $1 \mu \mathrm{g}$ methacholine which caused no significant increase in AHR above basal levels $(2.4 \pm$ 


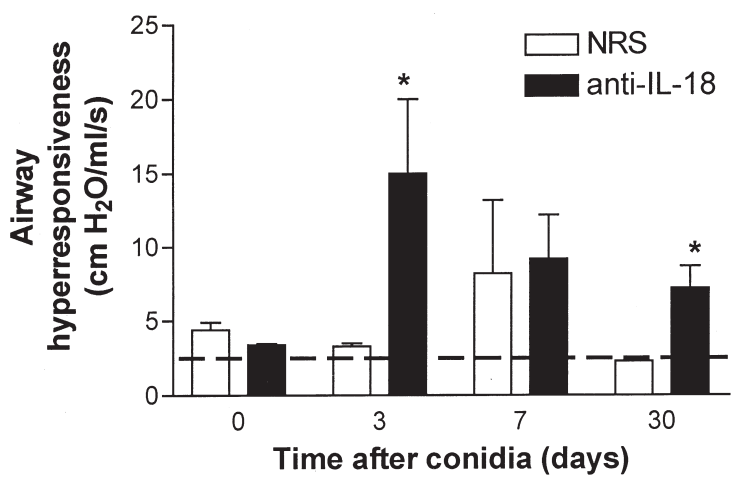

Fig. 4. Airway hyperresponsiveness in A. fumigatus-sensitized mice prior to, and at various times after, A. fumigatus conidia challenge. Changes in airway resistance or hyperresponsiveness (units $=\mathrm{cm}$ $\mathrm{H}_{2} \mathrm{O} / \mathrm{ml} / \mathrm{s}$ ) were monitored at each time point by the intravenous injection of methacholine in mice treated with NRS (open bars) or polyclonal anti-IL-18 antibodies (filled bars). The dashed line indicates airway resistance measured in both groups in the absence of methacholine (i.e. baseline resistance). Values are expressed as mean $\pm \mathrm{SEM}$; $\mathrm{n}=5-7$ /group/time point. $* \mathrm{P}<0.05$ denotes significant changes in airway resistance compared to NRS-treated control groups.

$0.5 \mathrm{~cm} \mathrm{H}_{2} \mathrm{O} / \mathrm{ml} / \mathrm{s}$; Fig. 4), was used. Immuno-neutralization of IL-18 in A. fumigatus-sensitized mice significantly $(\mathrm{P}<0.05)$ elevated AHR at day 3 and 30 after the conidia challenge compared to NRS-treated controls (Fig. 4). These data show that immunoneutralization of IL-18 accelerates and prolongs the extent of allergic airway hyperresponsiveness.

\section{A. fumigatus persistence within the lungs following $I L-18$ immunoneutralization}

Neutrophils play a key role in the removal of conidia following pulmonary infection. Since BAL neutrophil numbers were significantly decreased following IL-18 immunoneutralization, we examined whether $A$. fumigatus persisted in the lungs of these mice. The presence of fungus within the lungs was histologically examined using GMS stain, which appears black in the presence of the polysaccharide components from the fungus cell wall. Fig. 5a demonstrates the presence of few fungal conidia in the airways of NRS-treated control animals, however, a significant increase in staining was seen in anti-IL-18-treated mice (Fig. 5B). Although increased fungal staining was observed in these mice, there was no evidence of hyphal formation or fungal colonization. $\mathrm{H} \& \mathrm{E}$ staining of whole lung sections demonstrated granulomatous formations associated with the areas of fungal persistence in the lungs of anti-IL-18-treated (Fig. 5C), but not control mice (data not shown). Thus, the acute 3-day immunoneutralization of IL-18 results in the persistence of fungal conidia with the lungs thereby promoting the formation of granulomatous regions. This feature probably reflects the fact that neutrophil recruitment was impaired into the airways of anti-IL-18-treated mice.

\section{Evidence for chronic inflammation and airway remodeling following IL-18 neutralization}

Administration of live A. fumigatus conidia to mice previously sensitized to A. fumigatus has been shown to cause subepithelial/peribronchial fibrosis [16]. Increased collagen deposition and peribronchial fibrosis, a sign of chronic inflammation, is readily visualized by Masson Trichrome staining at various times following conidia challenge [16] (Fig. 6A). In the present study, the greatest staining was seen at day 30 after conidia in NRS-treated mice. However, in anti-IL-18-treated mice, subepithelial fibrosis was markedly increased above that seen in the NRS control group (Fig. 6B). The histological analysis suggested that IL-18 immunoneutralization lead to chronic and persistent airway remodeling.

\section{IL-18 regulates TLR 2 expression in A. fumigatus-sensitized mice during conidia challenge}

To further investigate the mechanism for conidia persistence within the lungs, we measured the levels of receptors involved in the innate immune response that are thought to recognize fungal components. TLR-2 is involved in inducing anti-fungal responses in Drosophila [19], and recent studies have indicated a role for these receptors in innate responses mediated by products of various infectious agents [20]. We examined the expression of TLR2 mRNA in whole lungs from non-sensitized mice receiving conidia alone or A. fumigatus-sensitized animals following IL-18 immunoneutralization to determine whether the expression of this receptor is modulated by IL-18. TLR2 mRNA was expressed constitutively in the lungs of non-sensitized mice prior to conidia challenge $(64.4 \pm 2.0 \beta$-actin/TLR2 ratio; Fig. 7A) and increased significantly at days $21 \quad(\mathrm{P}<0.05)$ and 30 $(\mathrm{P}<0.001)$ after the conidia challenge. In contrast, TLR2 mRNA expression was absent in mice previously sensitized to A. fumigatus prior to the conidia challenge (Fig. 7B) but was significantly elevated $3,7(\mathrm{P}<0.05)$ and $30(\mathrm{P}<0.001)$ days after conidia challenge in these mice (Fig. 7B). Interestingly, TLR2 mRNA levels induced after conidia challenge in the sensitized mice were approximately 10 fold lower than that observed in non-sensitized mice. IL-18 immunoneutralization further decreased the levels of TLR2 mRNA in whole lung samples after conidia challenge; TLR2 mRNA was not significantly $(\mathrm{P}<0.0001)$ elevated until 30 days after conidia challenge in mice that received IL-18 antibodies 0 to 3 days after conidia challenge (Fig. 7C). Taken together, these data suggest that $A$. fumigatus sensitization delays TLR2 mRNA upregulation and that anti-IL-18 treatment further delays TLR2 expression. The lack of TLR2 strongly correlates with the retention of conidia in the airway.

\section{Discussion}

Previous studies demonstrate that IL-12- and IL-18-induced IFN- $\gamma$ production is essential for the clearance of fungal infection [8]. However, in the case of allergic fungal disease, the initial, early Th1 response is followed by a vigorous Th2 response. At present, the role that IL-18 plays in the early 


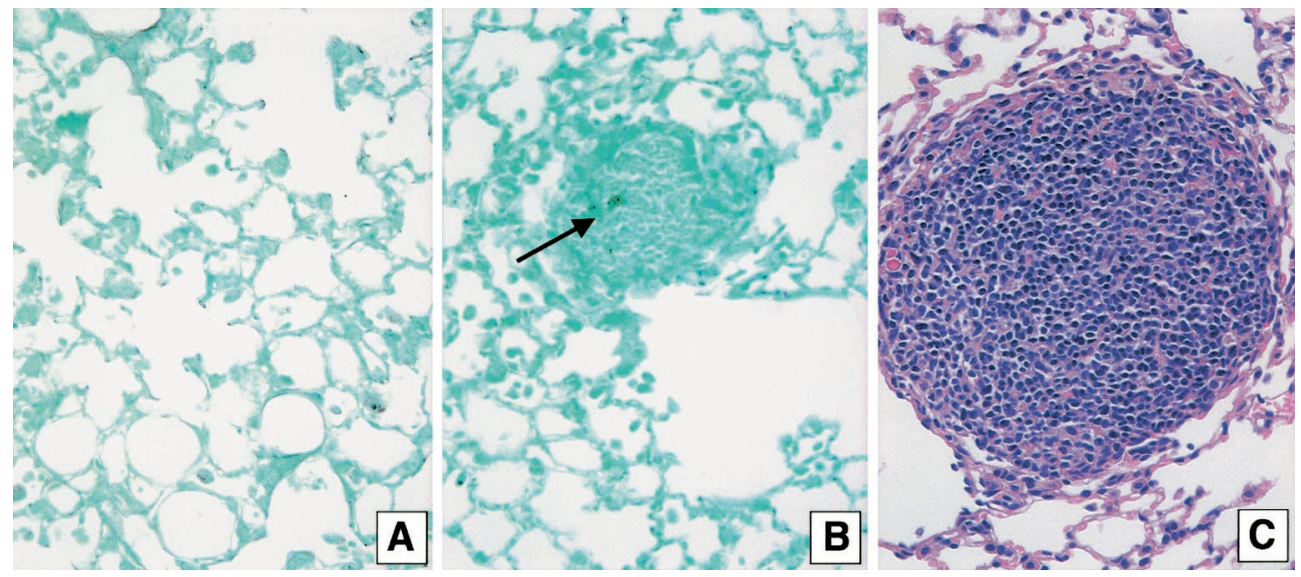

Fig. 5. Representative photomicrographs of GMS-stained whole lung sections from A. fumigatus-sensitized mice 30 days after conidia challenge. Mice were treated with NRS (A) or anti-IL-18 polyclonal antibodies $(\mathbf{B})$ between day $0-3$ after conidia challenge. GMS staining was evident in alveolar macrophages in whole lung sections from IL-18 depleted mice as shown by the arrow (B) but was absent in similar sections from NRS-treated control lungs (A). (C) shows a representative photomicrograph of hematoxylin and eosin-stained whole lung section from A. fumigatus-sensitized mice 30 days post conidia challenge treated with IL-18 antibodies showing granuloma formation within the lungs. Original magnification was $200 \times$.

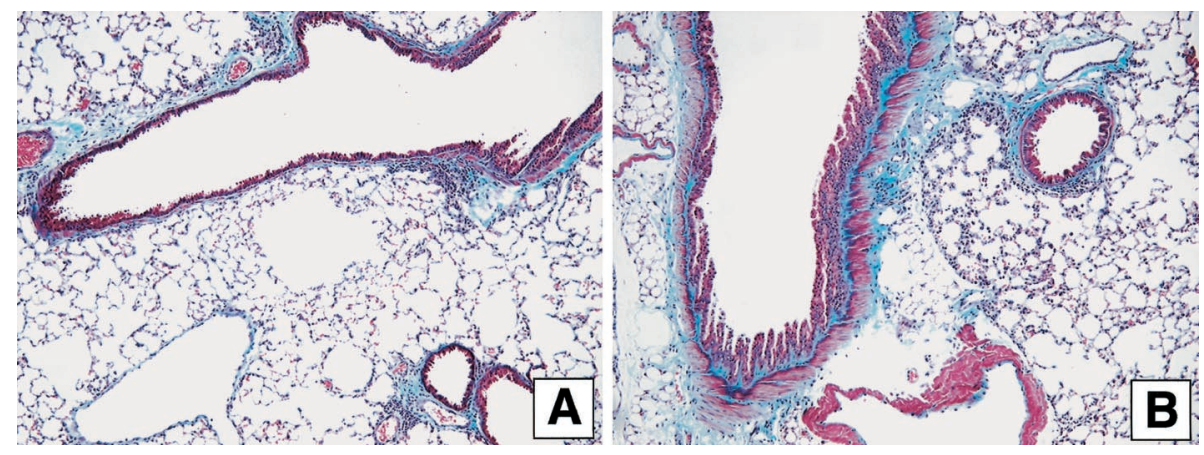

Fig. 6. Masson trichrome-stained whole lung sections 30 days after conidia challenge showing the extent of collagen deposition (light blue staining) around small airways and adjacent blood vessels. Sections were taken from A. fumigatus-sensitized mice treated with NRS (A) or anti-IL-18 antibodies (B) between day $0-3$ after conidia challenge. Greater amounts of collagen were apparent around the airways from mice treated with anti-IL18 antibodies compared with NRS. Original magnification was $200 \times$ for each photomicrograph.

Th1, or later Th2, response in fungal-induced asthma is unclear. The present study was designed to investigate the role of this cytokine during chronic development of allergic fungal disease. IL-18, IFN- $\gamma$, and IL-12 levels in whole lung homogenates were significantly elevated 3 days after conidia challenge in this model of fungal induced allergic asthma. Previous studies have shown that IL-18, released from alveolar macrophages or airway epithelial cells [21, 22], in concert with IL-12, induces IFN- $\gamma$ secretion from alveolar macrophages, T cells, and NK cells $[8,21,23]$. Since the coordinated expression of these Th1-type cytokines plays a key role in the defense against fungal colonization [8, 24], these observations suggest that IL-18 may regulate the initial host response to A. fumigatus conidia. The present study demonstrated that IL-18 expression subsequent to the intratracheal administration of live A. fumigatus conidia in $A$. fumigatus-sensitized mice plays a key role in the clearance of conidia from the lungs. The lack of fungus clearance from anti-IL-18-treated mice further compounded AHR, eosinophilic lung inflammation, and peribronchial fibrosis.
In agreement with previous studies, IL-18 immunoneutralization did not alter IL-12 or IFN- $\gamma$ levels in whole lung homogenates compared to control-treated animals $[18,25]$. IL-18 has previously been shown to synergize with IL-12 to induce the production of IFN- $\gamma$ from NK cells and macrophages via upregulation of the IL-12R $\alpha$ subunit $[8$, 23], however, it is unable to induce the release of IFN- $\gamma$ independently. While IL-18 does not alter IL-12 or IFN- $\gamma$ levels in whole lung homogenates, conidia clearance from the lungs of A. fumigatus-sensitized mice is clearly delayed following IL-18 immunoneutralization. This suggests that IL-18 has an integral role in the initial host immune response to fungal conidia that is independent of IFN- $y$ or IL-12 expression in the allergic host. Anti-IL-18 treatment was associated with significantly decreased neutrophil accumulation in the BAL at 3 days after conidia challenge in A. fumigatus-sensitized mice. Since neutrophils have been shown to be intimately involved in the clearance of conidia from the lungs [26], decreased neutrophil migration into the airways of anti-IL18 -treated mice probably allowed the retention of conidia. 
A

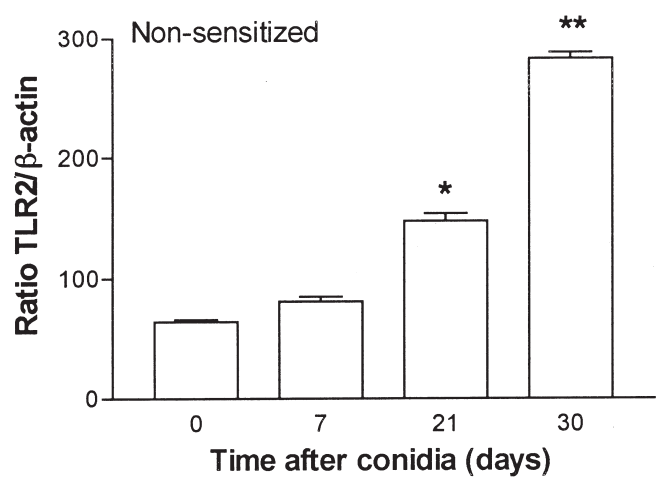

B

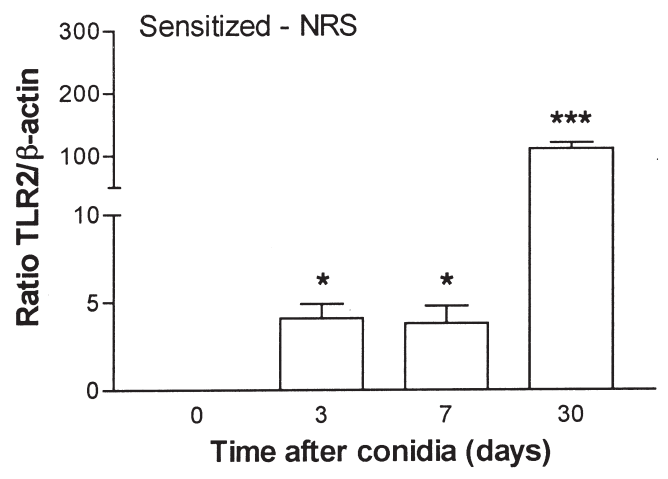

C

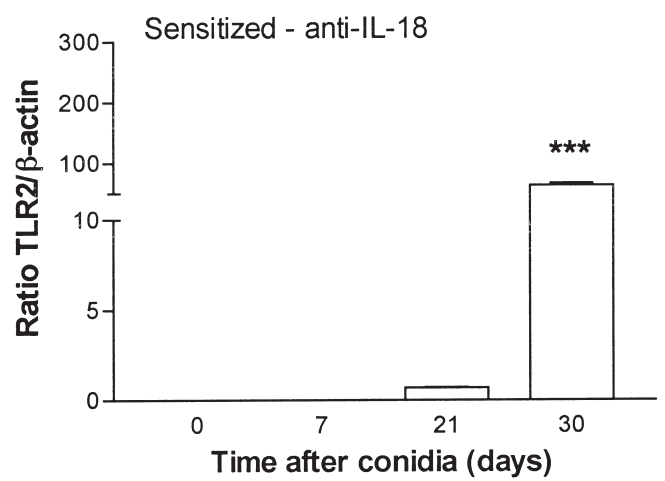

Fig. 7. Effects of anti-IL-18 treatment in non-sensitized mice (A), $A$. fumigatus-sensitized mice treated with NRS between days $0-3$ after conidia (B), and $A$. fumigatus-sensitized mice treated with anti-IL-18 anti-serum between days $0-3$ after conidia challenge (C). mRNA was isolated prior to, and 3,7, and 30 days after, conidia challenge. Changes in the levels of $\beta$-actin and TLR2 mRNA expression in whole lung homogenates were measured by semi-quantitative RT-PCR. Induction was expressed as a ratio of TLR $2 / \beta$-actin mRNA density. Data is expressed as means \pm SEM of TLR $2 / \beta$-actin ratios for 3 individual experiments. $* \mathrm{P}<0.05, * * \mathrm{P}<0.01$ and $* * * \mathrm{P}<0.0001$ denotes significant increases in TLR2 expression compared to levels measured prior to conidia challenge.
The decreased neutrophil tissue accumulation noted in this research coincides with a previous study by Netea et al [27] who showed that neutrophil recruitment in a murine model of Escherichia coli-induced endotoxemia was defective after anti-IL-18-treatement. They also suggested that this defect was mediated via several pathways involving decreased IFN- $\gamma$, macrophage inhibitory protein-2 (MIP-2), TNF- $\alpha$, and ICAM-1 expression. In the context of allergic airway disease, no significant differences in whole lung levels of IFN$\gamma$, TNF- $\alpha$, or MIP-2 were detected at 3 days following conidia challenge in anti-IL-18-treated mice compared with NRS-treated controls, but changes in the levels of these cytokines or chemokines may have occurred at earlier time points. In contrast to the decrease 3 days after conidia challenge, a significant increase in BALF neutrophil numbers was noted 30 days after conidia challenge. This observation may be a result of persistent conidia or subsequent pulmonary infections such as pneumonitis.

The increased AHR after conidia challenge in anti-IL-18treated mice correlated with increased peribronchial and BALF eosinophilia. Elevated airway hyperresponsiveness accompanied by BALF eosinophilia and elevated IgE levels have previously been described following neutralization of IL-18 or in IL-18 deficient murine models of acute allergic lung disease $[17,18]$. Campbell et al [17] showed that IL-18 immunoneutralization allergen-associated eosinophil accumulation could be induced $8 \mathrm{~h}$ after cockroach allergen challenge in mice previously sensitized to cockroach allergen. Furthermore, corroborating the results of the present study, Campbell et al [17] demonstrated an increase in AHR to methacholine following immunoneutralization of IL-18. Similarly, Kodama et al [18] demonstrated significantly higher levels of lung eosinophilia in IL-18 deficient mice in an ovalbumin model of allergic airway disease and demonstrate that, in vivo, IL-18 inhibited antigen-specific Th2 development. In contrast, administration of recombinant murine IL-18 has been shown to increase allergic sensitization, Th2 cytokine production, and airway eosinophilia in a ragweed model of murine allergic asthma [28]. This data suggests that IL-18 may promote a Th2 phenotype in vivo and potently induce allergic sensitization [28]. In the present study, AHR was significantly elevated 3 days after the conidia challenge in anti-IL-18-treated mice. Interestingly, AHR remained elevated at 30 days after conidia challenge in these mice. The increase in AHR in anti-IL-18-treated mice at day 30 after conidia challenge may have been a result of a persistent fungal antigen challenge and increased eosinophil numbers. In addition, profound peribronchial and interstitial remodeling were observed in the lungs of IL-18 depleted mice - all pathological features that are likely to contribute to elevated AHR responses observed at this time point $[29,30]$. Thus, increased AHR was a persistent feature of chronic fungal asthma following an acute, 3-day, immunoneutralization of IL-18.

The regulation of fibrosis and collagen deposition is a dynamic event, involving synthesis and deposition of collagen as well as degradation of existing basement membrane components. Collagen deposition, subepithelial fibrosis, and granuloma formation were markedly increased in the lungs of IL-18-depleted mice 30 days after conidia challenge compared with their NRS controls; these alterations could have 
been the consequence of a number of concurrent events. First, the elevated eosinophil numbers detected in the lungs of anti-IL-18-treated mice may have, in part, been responsible for the chronic structural changes observed in the airways. Support for a role for eosinophils in pulmonary fibrosis has been drawn from clinical data showing a correlation between lung eosinophil count and pulmonary fibrosis [31]. Furthermore, eosinophils are a major source for several key pro-fibrogenic cytokines such as TGF- $\beta$ and TNF- $\alpha$. In vitro studies that have shown that eosinophils, via elaboration of both cytokines, can stimulate fibroblast proliferation and contribute to profibrotic events [31]. Second, the release of matrix metalloproteinases (MMPs) may also play a key role in this interaction. MMPs are generally accepted as important mediators of proteolytic activity during many physiological and pathological remodeling processes [32], and the regulation of MMP release and activity appears to be altered in the lungs of asthmatics [33]. While there are no previous studies showing that IL-18 directly influences collagen deposition and subepithelial fibrosis, we investigated, in the present study, the role of MMP-2. Preliminary results in vitro suggest that IL-18 increases MMP-2 expression in alveolar macrophages, leading to the speculation that the lack of IL18 may have suppressed MMP-2 expression thereby leading to impaired degradation of collagen. However, we are currently investigating the modulation of MMP levels within the whole lung in a follow up study, as epithelial cells in addition to alveolar macrophages produce MMPs. Finally, increased airway remodeling 30 days after conidia challenge may have also been a consequence of delayed conidia clearance. Interestingly, the immunoneutralization of IL-18 in conidia-challenged mice provoked major interstitial remodeling as evidenced by granuloma formation. Sugawara et al [25] observed that larger granulomatous regions formed in the lungs of IL-18 deficient mice following Mycobacterium tuberculosis infection compared to wild type controls. These results reinforce those obtained in the present study, whereby immunoneutralization of IL-18 led to the formation of granulomas in the lungs of A. fumigatus-sensitized mice 30 days after conidia challenge, while no granulomas were observed in mice that received NRS during the conidia challenge.

The present study also addressed the impact of IL-18 on TLR2 expression during allergic airway disease. In addition to clearance of conidia by neutrophils in IL-18-depleted mice, fungal recognition via specific receptors on immune cells may have been altered in allergic mice. Toll-like receptors have been identified as key participants in the recognition of infectious agents and activation of the innate immune response. Toll was initially identified as a key protein in the development of the Drosophila body plan, however, later in the fly's life, this protein is involved in the defensive response to fungal infection [19]. Consistent with previous studies [34], constitutive TLR2 mRNA expression was observed in whole lung homogenates from non-sensitized mice prior to conidia challenge, while A. fumigatus-sensitization abrogated this constitutive TLR2 mRNA. Following conidia challenge, TLR2 mRNA expression is upregulated in both nonsensitized and A. fumigatus-sensitized mice - with much higher expression noted in non-sensitized mice. Delayed, or suppressed, TLR2 mRNA expression following A. fumigatus sensitization correlates with the delay in clearance of fungal conidia in these mice compared to non-sensitized mice. Further, TLR2 mRNA expression after conidia challenge in IL18-depleted mice is suppressed until day 30 after conidia, which correlates with the increased retention of conidia in the lungs of these mice. Decreased, or delayed, TLR2 expression appears to prevent efficient clearance of conidia from the lungs, and persistence of conidia in the lungs may lead to the onset of exacerbated allergic airway disease, fibrosis, and remodeling. The avalability of suitable reagents limits the study of TLR2's role in this disease, however, we await the accessibility of TLR2 knockout to fully investigate the role of TLR2 in fungal clearance and pulmonary fibrosis.

The results presented in this manuscript demonstrate the important role of IL-18 in the innate response to a conidia challenge in A. fumigatus-sensitized mice, possibly via the recruitment of phagocytic leukocytes such as neutrophils within the lung and regulation of recognition receptor expression (i.e. TLR2). Delayed fungal clearance in the absence of IL-18 impacted the development of allergic airway responses at later times points following conidia challenge, resulting in the exacerbation of AHR and dramatic airway and interstitial remodeling responses.

Acknowledgements. This study was supported by the National Institutes of Health Grants, HL31963, HLP50-5604 and HL35276.

\section{References}

[1] Greenberger PA, Patterson R. Allergic bronchopulmonary aspergillosis. Model of bronchopulmonary disease with defined serologic, radiologic, pathologic and clinical findings from asthma to fatal destructive lung disease. Chest 1987; 91(6 Suppl): $165 \mathrm{~S}-171 \mathrm{~S}$.

[2] Kauffman HF, Tomee JF, van der Werf TS, de Monchy JG, Koeter GK. Review of fungus-induced asthmatic reactions. Am J Respir Crit Care Med 1995; 151(6): 2109-15; discussion 2116.

[3] Cenci E, Mencacci A, Fe d'Ostiani C, Montagnoli C, Bacci A, Del Sero $\mathrm{G}$ et al. Cytokine- and T-helper-dependent immunity in murine aspergillosis. Res Immunol 1998; 149(4-5): 445-54; discussion 504-5.

[4] Cenci E, Perito S, Enssle KH, Mosci P, Latge JP, Romani L et al. Th1 and Th2 cytokines in mice with invasive aspergillosis. Infect Immun 1997; 65(2): 564-70.

[5] Chang JT, Segal BM, Nakanishi K, Okamura H, Shevach EM. The costimulatory effect of IL-18 on the induction of antigen-specific IFN-gamma production by resting T cells is IL-12 dependent and is mediated by up-regulation of the IL-12 receptor beta2 subunit. Eur J Immunol 2000; 30(4): 1113-9.

[6] Okamura H, Kashiwamura S, Tsutsui H, Yoshimoto T, Nakanishi K. Regulation of interferon-gamma production by IL-12 and IL18. Curr Opin Immunol 1998; 10(3): 259-64.

[7] Bohn E, Sing A, Zumbihl R, Bielfeldt C, Okamura H, Kurimoto M et al. IL-18 (IFN-gamma-inducing factor) regulates early cytokine production in, and promotes resolution of, bacterial infection in mice. J Immunol 1998; 160(1): 299-307.

[8] Zhang T, Kawakami K, Qureshi MH, Okamura H, Kurimoto M, Saito A. Interleukin-12 (IL-12) and IL-18 synergistically induce the fungicidal activity of murine peritoneal exudate cells against Cryptococcus neoformans through production of gamma interferon by natural killer cells. Infect Immun 1997; 65(9): 3594-9.

[9] Hogaboam CM, Gallinat CS, Taub DD, Strieter RM, Kunkel SL, Lukacs NW. Immunomodulatory role of $\mathrm{C} 10$ chemokine in a murine model of allergic bronchopulmonary aspergillosis. J Immunol 1999; 162(10): 6071-9. 
[10] Mehrad B, Strieter RM, Standiford TJ. Role of TNF-alpha in pulmonary host defense in murine invasive aspergillosis. J Immunol 1999; 162(3): 1633-40.

[11] Lukacs NW, Strieter RM, Warmington K, Lincoln P, Chensue SW, Kunkel SL. Differential recruitment of leukocyte populations and alteration of airway hyperreactivity by $\mathrm{C}-\mathrm{C}$ family chemokines in allergic airway inflammation. J Immunol 1997; 158(9): 4398-404.

[12] Evanoff HL, Burdick MD, Moore SA, Kunkel SL, Strieter RM. A sensitive ELISA for the detection of human monocyte chemoattractant protein-1 (MCP-1). Immunol Invest 1992; 21(1): 39-45.

[13] Kurup VP, Grunig G, Knutsen AP, Murali PS. Cytokines in allergic bronchopulmonary aspergillosis. Res Immunol 1998;149(45):466-77; discussion 515-6.

[14] Blease K, Mehrad B, Standiford TJ, Lukacs NW, Kunkel SL, Chensue SW et al. Airway Remodeling Is Absent in CCR1-/- Mice During Chronic Fungal Allergic Airway Disease. J Immunol 2000; 165(3): 1564-72.

[15] Blease K, Mehrad B, Standiford TJ, Lukacs NW, Gosling J, Boring $\mathrm{L}$ et al. Enhanced Pulmonary Allergic Responses to Aspergillus in CCR2-/- Mice. J Immunol 2000; 165(5): 2603-11.

[16] Hogaboam CM, Blease K, Mehrad B, Steinhauser ML, Standiford TJ, Kunkel SL et al. Chronic Airway Hyperreactivity, Goblet Cell Hyperplasia, and Peribronchial Fibrosis during Allergic Airway Disease Induced by Aspergillus fumigatus. Am J Pathol 2000; 156(2): 723-32.

[17] Campbell E, Kunkel SL, Strieter RM, Lukacs NW. Differential roles of IL-18 in allergic airway disease: induction of eotaxin by resident cell populations exacerbates eosinophil accumulation. J Immunol 2000; 164(2): 1096-102.

[18] Kodama T, Matsuyama T, Kuribayashi K, Nishioka Y, Sugita M, Akira $\mathrm{S}$ et al. IL-18 deficiency selectively enhances allergeninduced eosinophilia in mice. J Allergy Clin Immunol 2000; 105(1 Pt 1): 45-53.

[19] Lemaitre B, Nicolas E, Michaut L, Reichhart JM, Hoffmann JA. The dorsoventral regulatory gene cassette spatzle/Toll/cactus controls the potent antifungal response in Drosophila adults. Cell 1996; 86(6): 973-83.

[20] Yang RB, Mark MR, Gray A, Huang A, Xie MH, Zhang M et al. Toll-like receptor-2 mediates lipopolysaccharide-induced cellular signalling [see comments]. Nature 1998; 395(6699): 284-8.

[21] Cameron LA, Taha RA, Tsicopoulos A, Kurimoto M, Olivenstein R, Wallaert B et al. Airway epithelium expresses interleukin-18 [In Process Citation]. Eur Respir J 1999; 14(3): 553-9.

[22] Muneta Y, Shimoji Y, Yokomizo Y, Mori Y. Molecular cloning of porcine interleukin-1 beta converting enzyme and differential gene expression of IL-1beta converting enzyme, IL-1beta, and IL-18 in porcine alveolar macrophages. J Interferon Cytokine Res 1999; 19(11): 1289-96.

[23] Munder M, Mallo M, Eichmann K, Modolell M. Murine macrophages secrete interferon gamma upon combined stimulation with interleukin (IL)-12 and IL-18: A novel pathway of autocrine macrophage activation. J Exp Med 1998; 187(12): 2103-8.

[24] Kawakami K, Qureshi MH, Zhang T, Okamura H, Kurimoto M, Saito A. IL-18 protects mice against pulmonary and disseminated infection with Cryptococcus neoformans by inducing IFN-gamma production. J Immunol 1997; 159(11): 5528-34.

[25] Sugawara I, Yamada H, Kaneko H, Mizuno S, Takeda K, Akira S. Role of interleukin-18 (IL-18) in mycobacterial infection in IL-18gene- disrupted mice. Infect Immun 1999; 67(5): 2585-9.

[26] Latge JP. Aspergillus fumigatus and aspergillosis. Clin Microbiol Rev 1999; 12(2): 310-50.

[27] Netea MG, Fantuzzi G, Kullberg BJ, Stuyt RJ, Pulido EJ, McIntyre RC Jr. et al. Neutralization of IL-18 reduces neutrophil tissue accumulation and protects mice against lethal escherichia coli and salmonella typhimurium endotoxemia [In Process Citation]. J Immunol 2000; 164(5): 2644-9.

[28] Wild JS, Sigounas A, Sur N, Siddiqui MS, Alam R, Kurimoto M et al. IFN-gamma-inducing factor (IL-18) increases allergic sensitization, serum IgE, Th2 cytokines, and airway eosinophilia in a mouse model of allergic asthma [In Process Citation]. J Immunol 2000; 164(5): 2701-10.

[29] Bousquet J, Jeffery PK, Busse WW, Johnson M, Vignola AM. Asthma. From bronchoconstriction to airways inflammation and remodeling. Am J Respir Crit Care Med 2000; 161(5): 1720 45.

[30] Boulet LP, Laviolette M, Turcotte H, Cartier A, Dugas M, Malo JL et al. Bronchial subepithelial fibrosis correlates with airway responsiveness to methacholine. Chest 1997; 112(1): 45-52.

[31] Gharaee-Kermani M, Phan SH. The role of eosinophils in pulmonary fibrosis (Review). Int J Mol Med 1998; 1(1): 43-53.

[32] Kugler A. Matrix metalloproteinases and their inhibitors. Anticancer Res 1999; 19 (2C): 1589-92.

[33] Holgate ST. Asthma: a dynamic disease of inflammation and repair. Ciba Found Symp 1997; 206: 5-28; discussion 28-34, 106-10.

[34] Matsuguchi T, Takagi K, Musikacharoen T, Yoshikai Y. Gene expressions of lipopolysaccharide receptors, toll-like receptors 2 and 4 , are differently regulated in mouse $\mathrm{T}$ lymphocytes. Blood 2000; 95(4): 1378-85.

To access this journal online: http://www.birkhauser.ch 